\title{
Wind Action Phenomena Associated with Large-Span Bridges
}

\author{
Daniel C. Vaz, Raquel A.B. Almeida and \\ António R. Janeiro Borges \\ Additional information is available at the end of the chapter \\ http://dx.doi.org/10.5772/intechopen.73061
}

\begin{abstract}
In the past, the design of bridges over increasing distances was limited by construction techniques and, as always, by economics. As technological advances have turned possible cable-supported bridges of incredible spans, a new challenge has been added to the equation: that of withstanding the action of winds without developing undesirable dynamic responses. In this chapter, the several aerodynamic phenomena of relevance to long-span bridges are classified and discussed. This will interest both experts and non-experts in the field, thanks to the overview that is given. For certain cases, codes of practice recommend wind tunnel tests. The reader is introduced to these, as well as to numerical simulations, which are currently gaining increasing importance. Next, measures for attenuating susceptibility for undesirable dynamic responses are reviewed. The chapter ends with a discussion of the Vila Real Bridge deck section, based on wind tunnel tests and numerical simulations carried out by the authors: the aerodynamics was effectively improved with geometrically subtle modifications that were proposed and adopted still in the design phase.
\end{abstract}

Keywords: wind actions, aeroelasticity, bridge decks, wind tunnel tests, aerodynamics, attenuation measures

\section{Introduction}

\subsection{Relevance of bridge aerodynamics}

Bridges are essential to the expansion and development of societies. Having in mind that erection sites are all different, we see a wide variety of bridges and it can even be said that no bridge is equal to the other. There are types of bridges but each case is a case; bridges are not a mass-production item. Several constraints to the design are brought into play, which 
vary from place to place and over history, such as economics, construction means and time schedule, difficulties of construction, and architectural aesthetics. Technological advances have made possible the construction of bridges over ever-increasing spans using less mass of materials, which is of socioeconomic interest.

According to the system supporting the deck, bridges can be classified into pillar-supported, arch-supported, cable-stayed, and cable-suspended. In moving from the first to the last case in this list, and generally speaking, the main span increases and, as a result, the flexibility of the bridge. Modern materials, construction techniques, and more accurate design impart to present-day bridges high flexibility and low structural damping, which are precisely the characteristics that render them more prone to aerodynamic instabilities. Indeed, the frequencies of the natural vibration modes of such bridges are sufficiently low to be within the energetic range of frequencies of the aerodynamic phenomena and thus undesirable resonance can take place.

When the structure vibrates, as a result of the fluid motion, its movement, if of sufficient amplitude, changes the flow pattern in the immediate vicinity of the structure, which in turn changes the effect of the flow on the structure. This results in a complex and dynamic interaction, called aeroelastic interaction, that does not correspond whatsoever to the situation in which the structure remains static or quasi-static.

Bridge design should bear in mind aeroelastic phenomena, in order to keep them safe for use, that is to say, under the action of wind, bridges shall not develop dangerous or inconvenient oscillations. In this regard, the deck's cross-section geometry is of paramount importance. Since it is not imposed by aerodynamics alone, such a thing as a single optimal type of deck is not available or possible, and instead there are a number of types of decks.

To show the relevance of the study of the aerodynamics of bridge decks, we give here three examples in which aerodynamic oscillations developed and had impact in serviceability or led to modification costs.

The Rio-Niterói Bridge (Rio de Janeiro, Brazil) has been the world's longest steel-box-girder pillarsupported bridge with a central span of $300 \mathrm{~m}$ at $72 \mathrm{~m}$ high. Since it opened to traffic in 1974, lowvelocity winds have often set it into oscillations [1]. When this happened, the bridge was closed to traffic, for the sake of user comfort and overall safety, though with important traffic inconveniences.

The Great Belt Suspension Bridge (Denmark) is, outside of Asia, the bridge with the longest main span: $1624 \mathrm{~m}$. It opened to traffic in 1998. During the final phases of construction, low-frequency vertical oscillations of the girder were observed for lateral wind of moderate speeds $(5-10 \mathrm{~m} / \mathrm{s})$ [2]. A theoretical study showed that the structural impact of the oscillations would be insignificant. However, there were concerns that the oscillations could distract drivers due to visual impact, jeopardise road safety, and convey a sense of unsafeness to the public potentially resulting in a loss in traffic volume. The oscillations were later attenuated with guide vanes mounted at the bottom edges of the girder.

More recently, in May 2010, months after being opened to traffic, the Volgograd Bridge (Russia) was closed to motor traffic for 5 days due to strong oscillations. In the autumn of 2011, this prestressed concrete girder bridge, with a main span of $160 \mathrm{~m}$, was fitted with tuned mass dampers to correct the situation [3]. 
This chapter is aimed at readers without expert knowledge in the field of aerodynamics and the objective is to provide information that the reader can later complete by exploring in more depth the available wealth of data in the published material.

\subsection{Typical bridge deck sections}

While, from the functional standpoint, a bridge deck is the indispensable element of a bridge, it is also the one that is of greater concern from the point of view of the bridge's aerodynamic performance. Its shape is of paramount importance and the design has to go hand in hand with structural criteria from civil engineering and the other aforementioned criteria. Consequently, a few types of decks exist. To begin with, we can distinguish between road and railway bridges. The latter are reinforced to increase the stiffness both in torsion and in bending. Long road bridges often have wider decks in order to accommodate more lanes of traffic. In what regards wind action upon them, one can identify decks with a continuous solid surface exposed to the lateral wind, like plate-girder, box-girder, and streamlined-trapezoidal deck sections, and decks based on trusses, which are permeable to the wind (Figure 1).

Each type of deck exhibits distinct susceptibility to aerodynamic phenomena. Moreover, for each one, several instances of aerodynamic studies can be found in the literature for the reason that decks of very similar geometry may nonetheless exhibit considerably distinct aerodynamic performances, be it as a result of the complexity of the aerodynamic phenomena or as a result of the dissimilarity between the dynamic properties of the decks. For example, for the same overall shape, a box-girder for a cable-stayed bridge with a central plane of cables has a

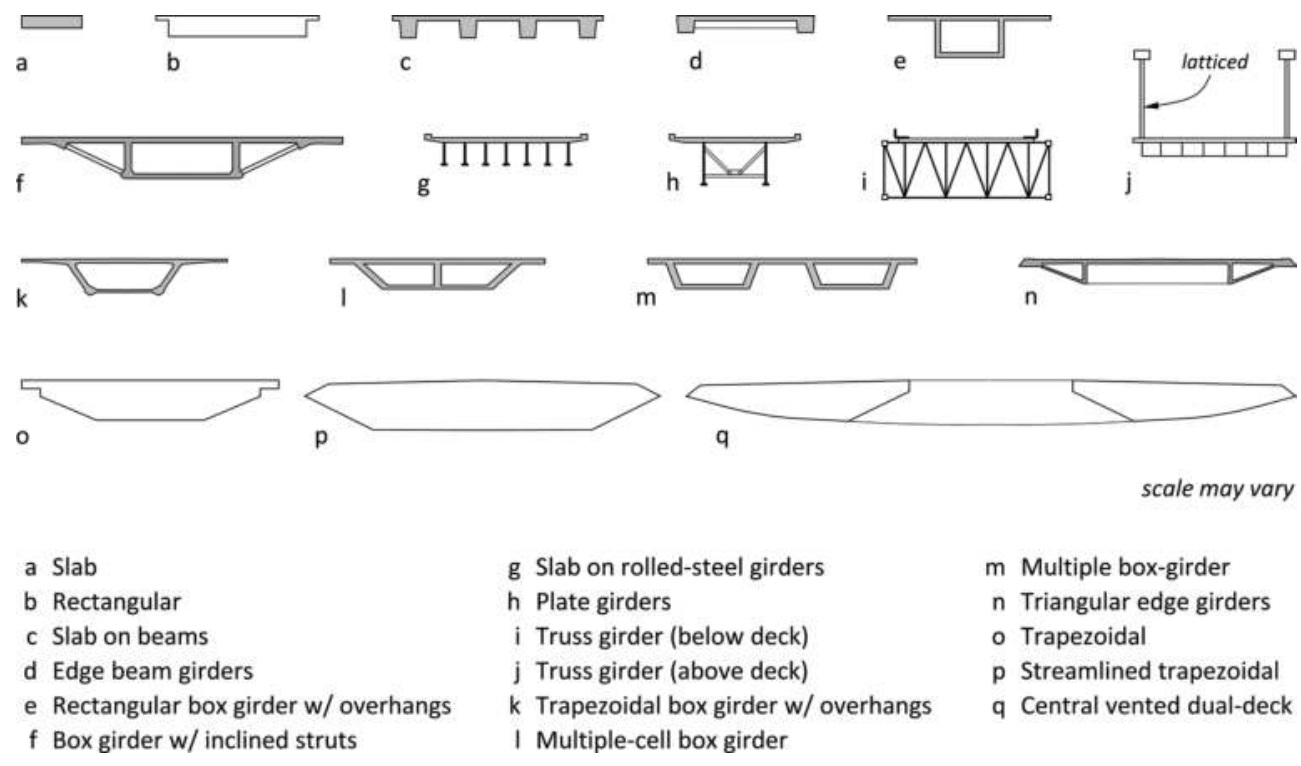

Figure 1. A selection of types of bridge decks. 


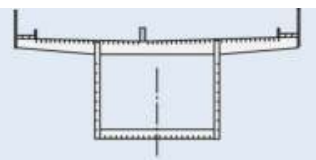

Europe, 1964

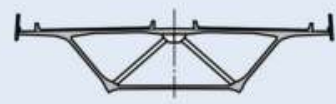

Brotonne, 1977

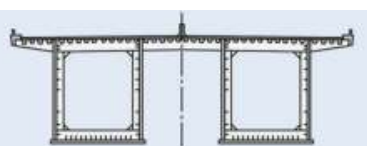

Niterói, 1974

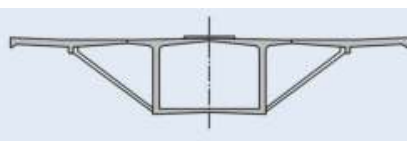

Eschachtal, 1977

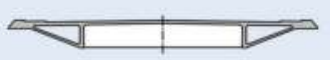

Pasco-Kennewick, 1978

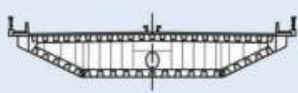

Farø South, 1985

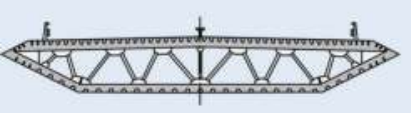

Great Belt East, 1998

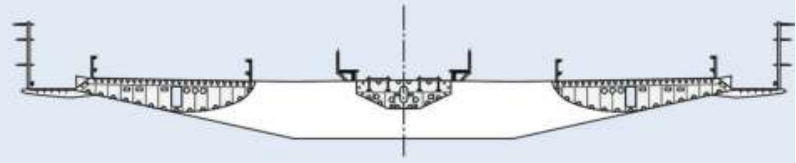

$10 \mathrm{~m} \quad$ Messina (approved 2011)

Figure 2. From general rectangular decks to trapezoidal and streamlined decks.

higher depth-to-width ratio than one for a bridge with a double plane of cables, because the girder rigidity has to compensate the lack of torsional stiffness in the first case. This difference in aspect ratio can lead to very distinct aerodynamic responses.

In box-girder decks, the quest for high aerodynamic performance has resulted in the shift from thick rectangular decks to trapezoidal and streamlined decks (Figure 2).

\section{Aerodynamic phenomena}

Wind action on bridges can adversely influence their static and dynamic stability. Alongwind deflections are examples of static reactions. These are minor problems in the sense that the existing codes of practice cover with sufficient detail current geometries of decks allowing conservative calculations. The same is not true with regards to the dynamic stability of large and very large-span bridges, which is related with vibrations or oscillations of the deck as a result of fluctuations in the pressure distribution over its surface. For very large-span bridges, a customised approach is mandatory, both analytical and experimental. The collapse of the Tacoma Narrows suspension bridge in 1940 alerted the scientific community to the importance of aerodynamic studies of wind actions on structures and to the possible occurrence of aeroelastic phenomena.

\subsection{Classification}

Several types of aerodynamic phenomena can develop as a consequence of interaction of actual wind with bridge decks. The turbulence of natural wind will always induce a fluctuating force 
on structures. This is known as buffeting and the associated displacements are usually of small amplitude, in a way that does not change the topology of the flow around the deck. In other situations, called aeroelastic phenomena, the oscillation amplitude is large enough to interfere with the airflow. In this case, the flow pattern around a deck is affected by the structure's motion itself, giving rise to a very complex interaction between the deck's motion and aerodynamic forces.

The flexibility of long-span bridges renders them highly susceptible to aeroelastic phenomena. In this context, vortex-induced vibration (VIV), torsional flutter, coupled flutter, and galloping may arise. While in VIV, the amplitude is self-limited, in the other three phenomena, the amplitude of the deck's motion tends to increase continuously and, for this reason, they are categorised as aeroelastic instabilities. If damping is insufficient, the large amplitude of the motion may cause collapse of the structure. Figure 3 systematises the classification of flow-induced vibrations on structures.

VIV may occur at low wind speeds, and when it does occur, it is for narrow ranges of wind speed specific for a given structure (Figure 4). Flutter-type instabilities arise at much higher wind speeds, above a critical value. In buffeting, turbulence in the incoming flow (a large band excitation) causes a response proportional to the dynamic pressure of the wind. The next sections address in more detail each type of flow-induced vibration.

\subsubsection{Buffeting}

As previously mentioned, buffeting refers to structural oscillations due to the turbulent fluctuations of oncoming wind. The structure can be considered absolutely rigid since the displacements are of very small amplitude. More specifically, the amplitude is much smaller than the thickness of the boundary layer ${ }^{1}$, and thus the vibration of the structure does not disrupt the boundary layer, thus not changing the topology of the flow around the deck.

Turbulence has a stochastic nature; pressure and velocity are random. The turbulence energy of natural wind concentrates in the lower frequency of the velocity spectrum. That is to say that if the characteristic lateral length of the structure is comparatively small (up to about $10^{2} \mathrm{~m}$ ),

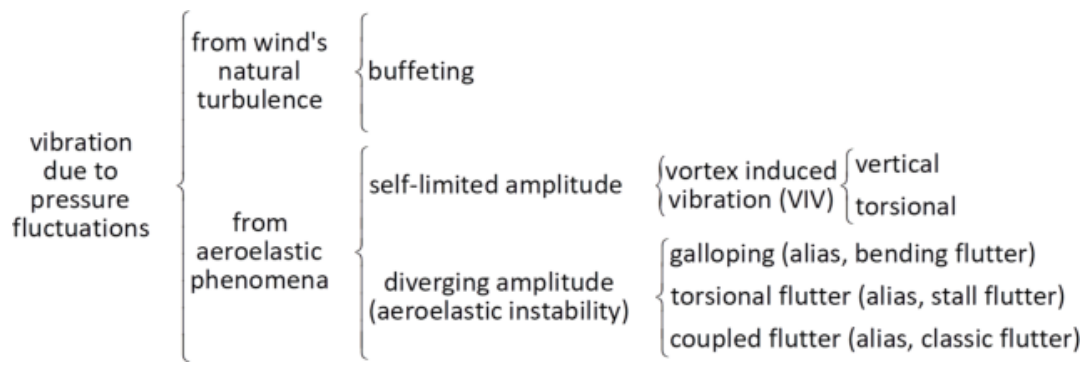

Figure 3. A classification of flow-induced vibrations, including aeroelastic phenomena.

${ }^{1}$ Boundary layer is the thin region near a solid surface where viscous effects are dominant and fluid velocity evolves from zero at the surface to nearly its free-stream value away from it. The depth of this layer is known as its thickness. 


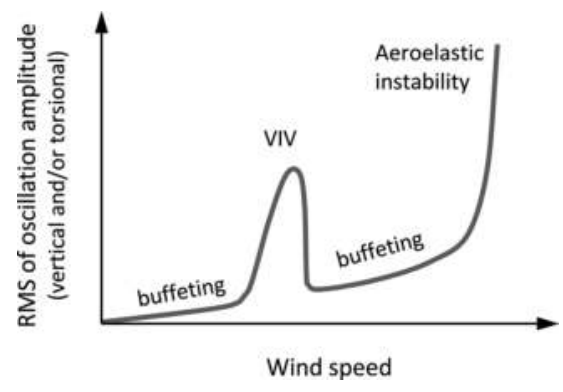

Figure 4. A generic graph of the relative amplitudes of the various flow-induced vibrations. Adapted from [4].

the aerodynamic force resulting from the turbulent flow acts as a spatially uniform fluctuating force; otherwise, the fluctuating wind can no longer be considered spatially uniform since the length scale of turbulence in the span-wise direction is comparable to, or smaller than, the span of the bridge. In this latter case, the wind peak speed effect is reduced. Indeed, the deck is not simultaneously excited over the whole of its length with the peak value of wind fluctuation; there are spatial and temporal variations.

Therefore, to optimise the dimensioning of the structure, instead of the wind peak speed, a reduced value should be used in the design. This entails the longitudinal cross-correlation of turbulent fluctuations. The problem is rather complex but the accumulated data concerning wind statistical properties and the development of analytical models associated with numerical techniques make it possible to tackle the influence of the three-dimensionality of natural wind fluctuations. From this, it is possible to derive reduction factors having in mind the relation between bridge span and turbulence length scale. If the spatial dimension of the correlation is considerably smaller than the bridge span, then the reduction factor will have a small value. Several examples of this procedure applied to bridges are available in the literature, and an interesting one concerns the Lion's Gate Bridge in Vancouver, Canada [5]. This analytical approach was developed to address the effect of gusts on aircraft wings and was transposed to civil engineering by Davenport [6].

Buffeting is proportional to the flow's dynamic pressure and thus the amplitude of buffetingrelated vibration exhibits a quadratic evolution with the flow velocity. In severe cases, it can destabilise vehicles or pedestrians. On the other hand, minor vibrations, which may go unnoticed, may cause in the long-term damage by fatigue to structural components.

Even bridges with a carefully designed cross-section of the deck can still be susceptible to buffeting. Buffeting may also stem from irregular flow separations along the contour of the bridge deck, which generate a new vortex field to be added to that of the oncoming turbulence [7]. Nevertheless, if the fluctuations associated with those separations lose their stochastic nature, and periodical vortical structures are formed in the wake, then this may turn out to be what is called vortex-shedding, to be dealt with in the next section. Buffeting refers also to a different effect: increased turbulence in the wake of a structure interfering with a nearby second one placed downwind (as in the l'Iroise Bridges), and the term wake-buffeting may be used in this case (Section 6.3 .3 of [8]). 


\subsubsection{Vortex-induced vibration}

Vortex-induced vibration (VIV) can appear as either vertical or torsional oscillations. It is caused when the flow separates at spots on the deck's contour so that vortices are shed periodically from one and the other sides of the bridge deck. Flow may separate at protuberances on the upper or lower surfaces of the deck, like traffic barriers or guard-rails. A detailed discussion of the flow physics of vortex-shedding excitation can be found in two recent reviews [7, 9].

When studying the aerodynamics of the Vila-Real Bridge, the authors concluded that VIV would not occur when vortices were shed from just one side of the deck [10]. The addition of road equipment to the top of the deck raised the possibility of vortex-shedding above the deck, in addition to occurrences on the other side.

The nature of the resulting aerodynamic forces acting on the deck makes VIV self-limited in amplitude.

Vortex-induced vibrations can cause discomfort to the users of a bridge or even its failure by fatigue in the long term. It should be pointed out that the amplitude can become of concern in situations for which the system has low damping and the vortex shedding frequency is close to a natural frequency of the bridge.

Lock-in is an interesting phenomenon associated to VIV, in which the high amplitude of the oscillating motion is maintained over a somewhat wider range of wind speeds rather than for a certain wind speed.

Decks known to be particularly susceptible to VIV are plate-girder and deep box-girder decks.

\subsubsection{Torsional flutter}

Torsional flutter, sometimes called stall flutter, happens when the damping associated with torsional motion is very low. Here, damping is to be understood as the ensemble of structural and aerodynamic damping of the structure acted by wind. A critical velocity can be defined as the value for which the total damping becomes zero. In the onset of torsional flutter, lift causes the body to vary its pitch in a way that from its interaction with the wind a sustained torsional motion results. Once established, torsional flutter manifests as a rapid rotational oscillation.

It should be said that static deformations induced by wind loadings at velocities near flutter occurrence may possibly change the effective wind attack angle.

Torsional flutter can occur, in occasions, when propitious conditions are met, in flexible bridges with channel type or H-shaped plate girder cross-sections.

\subsubsection{Galloping}

Galloping, also known as bending flutter, is a single degree-of-freedom aeroelastic oscillation in the direction transverse to the wind, which can reach large amplitude. There is potential for galloping to happen whenever $C_{L}(\alpha)$ exhibits an accentuated negative slope. Indeed, the total damping of a bluff body undergoing wind-excited oscillations consists of a structural 
damping (considered positive) and an aerodynamic damping, related to $d C_{L} / d \alpha+C_{D}$. The system can develop divergent oscillations if the total damping becomes negative. The condition for negative aerodynamic damping to occur is known as the Den Hartog criterion [11]. Galloping occurs above this critical wind speed.

Non-streamlined box-girders with relatively small width-to-depth ratio are particularly susceptible to galloping, since the flexural stiffness is much smaller than the torsional.

Galloping can occur at low, either steady or unsteady, wind speeds, for structures with low structural damping associated with bending, which is the case of suspension bridges, although the occurrence of galloping is more typical of overhead power lines subjected to ice deposition [11].

\subsubsection{Coupled flutter}

Coupled flutter, also named classic flutter, encompasses motion in both bending and torsion. It occurs in cases for which the structure has a pair of neighbouring frequencies in bending and in torsion.

Bridge decks that will exhibit coupled flutter if the wind speed exceeds a critical value are those with a streamlined trapezoidal cross-section, with large width-to-depth ratio, so that there are no large vortices shedding from the leading edge. Examples are the Great Belt and the Izmit Suspension Bridges, as pointed out by [7].

Classic flutter was first perceived in aircraft wings and Theodersen [12] developed a thin airfoil theory aimed at describing analytically the flutter phenomenon. Since the aerodynamics of trapezoidal decks is far from being tractable as that of a thin airfoil, this theory cannot be directly applied to bridge decks without corrections [13]. Scanlan and Sabzevari [14] proposed a formulation in which the aeroelastic forces (lift and torque) are expressed as functions involving coefficients known as flutter derivatives. These derivatives (which cannot be obtained analytically) have to be obtained through wind tunnel testing. The resulting equations can be solved for the critical wind speed for the onset of flutter.

In certain cases, designers may resort to simpler ways of estimating this critical speed. In the beginning of the 1960s, A. Selberg proposed a formula for thin plates. Under certain conditions, it can be adapted to bridge decks through the use of a factor that depends on the geometry of the cross-section. More recently, Bartoli and Mannini [15] proposed simple expressions for critically reduced wind speed and coupling frequency based on a reduced number of flutter derivatives.

\subsubsection{Comments}

Current design practice avoids coupled flutter, alias classic flutter, by having in mind from the beginning of the design sufficient separation between the lowest torsional natural frequency of the bridge and the fundamental bending frequency. For example, Section E.4.2 of the Eurocode 1 [8] establishes a minimum ratio of 2:1.

In the same way, torsional flutter is not a significant phenomenon in modern bridges due to the necessary stiffness imposed by practical reasons. 
Galloping can occur if Den Hartog's criterion is not observed. Vaz et al. [16] numerically studied two decks that were found stable. Nevertheless, galloping can be an important phenomenon in bridge cables, especially when the section's geometry may be changed by ice deposition.

From what has been said, it is understood that, as far as bridges are concerned, VIV is the aeroelastic phenomena that deserves the most attention. It is a major concern since the 1940s, when large oscillations began to be observed in the original Tacoma Narrows Bridge, at relatively low wind speeds.

\subsection{Influence of bridge features}

The occurrence of aeroelastic phenomena on long-span bridges has been widely studied. Good starting points for further reading are the reviews of Miyata [17] and of Saito and Sakata [18].

In aerodynamics, minor details (from the point of view of design) in the body contour can lead to significant modifications of the flow pattern and aerodynamic response. While this means that the aerodynamic can be improved by introducing, still at the design stage, inexpensive and simple-to-implement modifications, it also means that it is important to consider in the aerodynamic study the various stages of the deck construction since the addition of equipment, such as median dividers, guard rails, and border beams, can noticeably change the deck's aerodynamic response [17, 19-22].

\subsubsection{Deck cross-section shape}

The overall shape of the bridge deck's cross-section strongly influences the occurrence of aeroelastic phenomena [23-25]. A numerical study of four generic cross-section shapes developed from the well-known plate girder section of the First Tacoma Narrows Bridge, by adding horizontal plates and fairings, showed that the closed section with fairings displayed the best aerodynamic performance (in terms of drag loading and oscillating lift) and the parent H-shaped cross-section, the worst [23].

In streamlined box sections, VIV can likely be avoided or kept at insignificant levels if the angle between the horizontal extension of the deck's bottom plate and the lower inclined panels is kept smaller than $15^{\circ}$, in order to avoid flow separation [25]. Streamlining of the deck can possibly result in improved aerodynamic performance, with an increase in the critical wind speed for torsional flutter and decrease in the vortex-induced response [19, 26]. Even though streamlined decks have lower drag and postpone the onset of possible divergent aeroelastic instabilities to higher wind speeds, they may nevertheless exhibit oscillations at low wind speed due to vortex shedding excitation.

It should be said that trapezoidal decks are far from being tractable as thin airfoils and analytical methods, such as Theodersen's thin airfoil theory for the study of flutter, are not successful if applied without appropriate modifications. Bridge decks have to be considered as bluff bodies.

The aerodynamic stability of super-long-span bridges can be effectively improved by introducing a slot at the centre of the girder [24]. This is the design being adopted for the proposed Messina Strait (Figure 2) and Gibraltar Strait crossings [27, 28]. 


\subsubsection{Railings}

Changes in the porosity of the outermost railings, be it through the use of panels to deflect the wind (and thus improve the comfort of pedestrians or servicing personal) or by snow accumulation [29], would seem, at first glance, inconsequential for the bridge aerodynamics. However, making that area impermeable to the wind can render the deck section highly unstable. As the solidity ratio (considered high above 0.3 ) of edge safety barriers increases, so does the overall bluffness of the section, increasing drag and reducing mean lift; the effect is more relevant as the bare deck shape is more streamlined [20]. Decks with higher porosity railings have higher flutter critical wind speed [21]. The effect of the barriers contributed to the mechanism responsible for the vortex-induced oscillations of the Great Belt East Bridge in 1998 [20].

Deck equipment, such as median dividers, edge safety barriers, or parapets, can have a great impact on the bridge aerodynamics. In numerical simulations, the barriers should be included despite the increased computational effort, in order to take into account their effects on the flow [20].

\subsubsection{Supporting cables}

Cables are essential components of long-span bridges and they present small mass, higher flexibility in comparison to other bridge components, and low mechanical damping. Therefore, cables are even more prone to vibration than the deck.

Wind-induced flutter instability is a major concern in the design and construction of superlong-span cable-stayed bridges. While the aerodynamic contribution from the cables is generally despised when resorting to sectional models in wind tunnel experiments of cable-stayed bridges, this cannot be maintained when assessing super-long-span cable-stayed bridges. The influence of cable aerodynamic forces on the deck's flutter instability may be significant when the main span exceeds $1000 \mathrm{~m}$ and the frontal area (as viewed in the flow direction) of all stay cables exceeds that of the bridge deck.

\section{Codes of practice}

Due to the importance of the assessment of the response of bridges to the action of wind, codes of practice have been developed to aid the bridge engineer in the design calculations. They provide definitions, establish best practices, and give recommendations.

Most countries and regions of the world have their own codes of practice for bridge design, for example: "EN 1991, Eurocode 1: Actions on Structures" [8] in Europe; the "Specifications for Highway Bridges" by the Japan Road Association [30] or the "Design Standards of Superstructures for Long-Span Bridges" by the Honshu-Shikoku Bridge Authority [31], in Japan; the "LRFD Bridge Design Specifications" by the American Association of State Highway and Transportation Officials [32] in the USA; the "Wind-Resistant Design Specification for Highway Bridges" [33], in China; the "CAN/CSA-S6-00, Canadian Highway Bridge Design Code" [34], in Canada; and the Australian standard "AS 5100" [35], in Australia. 
In the European Union, the named Eurocodes ultimately replaced the several National Codes, with differing rules, in the various Member States. It is worth mentioning the recommendations of the European Convention for Constructional Steelwork (ECCS) [13] or, in the case of the UK up to 2010, the British standard "BS 5400" [36].

Wind actions on bridges are covered in Section 8 of the Eurocode 1 Part 1-4. It is worth pointing out that the code does not apply to bridges involving civil engineering works with heights above $200 \mathrm{~m}$ or bridges having spans greater than $200 \mathrm{~m}$. The Vila-Real Bridge to be discussed in Section 8 exceeds both these criteria.

Codes of practice tend to be conservative though, encouraging wind tunnel tests to address the susceptibility to undesirable aerodynamic phenomena, of long-span bridges and/or with atypical shape. In their numerical studies, Bruno and Mancini [20] found for decks with complex geometries errors up to $200 \%$ between the simulated forces and the predictions using standard rules (ENV 1991-2-4). Therefore, when it is difficult to fit up a bridge within the regulation in effect, it is unavoidable to resort to aerodynamic experimental studies to obtain design criteria that warrant structural safety and, in many cases, a reduction of the overall cost of the project.

\section{Wind tunnel testing}

\subsection{Introductory notes}

It should be said that wind tunnel tests will not directly provide, as an outcome, a design of a bridge or of an optimal deck geometry non-susceptible to aerodynamic instabilities. What the design engineer can expect from wind tunnel tests is a confirmation that the design is good from the aerodynamics point of view or, otherwise, clues that will help him/her in finding the causes of oscillations and remedy them. Nowadays, it is possible, and even advisable, to complement wind tunnel tests with numerical simulations, which are addressed in the next section.

Given the discussion in the preceding sections, it is not surprising that wind tunnel testing is an integral part of the design and analysis of most long-span bridges and is often a requirement in many codes and national standards. All over the world, many laboratories equipped with wind tunnels have been committed to such studies. In these studies, the goal is to reproduce, at a reduced scale and in the best possible way, the full-scale situation in a wind tunnel and to address whether the wind action on the bridge can possibly excite any of the bridge's vibration modes. When such excitation is possible to be foreseen, then it is necessary to propose corrective modifications and test their effectiveness in the wind tunnel.

\subsection{Similitude parameters}

Confidence in the translation to the real prototype of the results obtained with the wind tunnel models for the dynamic behaviour of bridge deck models imposes the compliance of certain similarity criteria. In what follows, the subscripts $m$ and $p$ refer to the model and to the prototype, respectively. 
The most natural criterion is geometric similarity, being expressed by the equation:

$$
L_{p} / L_{m}=C
$$

where $C$ is a constant that establishes the scale factor between the model and prototype. Geometric similarity implies the homothety of the shapes of the surfaces contacting with the air, excluding of course all the details that are of very difficult construction and not significant, or even completely irrelevant given the precision with which the parameters of interest are measured.

Kinematic similarity implies that the relation between the characteristic velocities of the flow over the model, $V_{m^{\prime}}$ and of the oscillatory movement of the model, $f_{z} L_{m^{\prime}}$ is the same for the prototype. This leads to the definition of a non-dimensional parameter known as reduced velocity:

$$
V_{r}=\frac{V}{f L}
$$

Here, $f$ is one of the structure's eigen-frequencies, and it is often the frequency of the fundamental vertical motion, $f_{z}$. Then, the relation between kinematically equivalent velocity scales between the prototype and the model can be defined as:

$$
\frac{V_{p}}{V_{m}}=C \frac{f_{z, p}}{f_{z, m}}
$$

The ratio of frequencies in (3) constitutes a relation for kinematically equivalent time scales, $T_{m} / T_{p^{\prime}}$ which is useful to estimate the sampling time interval in order to obtain the mean values of the parameters measured in the laboratory.

Dynamic similarity concerns the dynamic interaction between a body and the flow around it and is of a rather complex nature and thus more difficult to assure. In order to be observed, the relative magnitudes of the various forces involved in the structure's dynamics - the inertial, gravitational, aerodynamic, elastic, and structural damping forces-shall be the same for the model and the prototype, and, consequently, the motion amplitudes will be in the same proportion as the geometric scale ratio. For the airflow, the relation between inertial and viscous forces is expressed by the Reynolds number:

$$
\operatorname{Re}=V L / v
$$

Since the values of the kinematic viscosity of air, $v$, are approximately equal in the model and in the prototype, dynamic similarity would require that $V_{m}=C V_{p}$. This is in conflict with relation (3) established by kinematic similarity, which states that flow velocities in the wind tunnel represent much higher velocities of the flow over the prototype. However, when the model does not exhibit a streamlined section, recirculation zones are formed from flow detachments occurring at the model's sharp edges. This type of separation of the main flow from the solid surface is known to be independent of Re above a certain value. Therefore, it is important to warrant that the scale of the model and velocity in the wind tunnel result in a value of $R e$ above that threshold. 
The similarity parameter that involves the mass of the model, or of the prototype, together with the respective logarithmic decrements of the damped responses in the various oscillation modes remains to be discussed. Strictly, these variables should be addressed separately. However, when the main concern of wind tunnel studies is the analysis of vortex shedding, mass and damping can be brought together into a single non-dimensional parameter. That is to say, the system's response to the forced periodic excitation due to vortex shedding can be represented by the logarithmic damping factor, $\delta_{s^{\prime}}$ which is itself non-dimensional. This is appropriate in situations of low damping linear systems, and in such cases, the amplitudes of the resonance peaks are inversely proportional to damping. The similarity parameter, which is named Scruton number, is defined by the following expression [8]:

$$
S c=\frac{2 \delta_{s} m_{i}}{\rho h^{2}}
$$

where $m_{i}$ is a mass per unit length, $\rho$ is the air density, and $h$ is the cross-wind dimension (i.e., the structure's depth, specifically the section at which resonant vortex shedding occurs). Thus, the denominator in the definition represents a characteristic value of the mass of the air displaced by the structure. The Scruton number describes how sensitive a structure is to vibrating as a result of vortex shedding; the response amplitude will significantly increase at low values of this parameter.

Being extremely difficult in practice to build a model and an experimental apparatus leading to the same values of $S c$ in the model and prototype, the common practice is to build a model and suspension system as light and as least damped as possible, in order to bring out in the laboratory any tendency for instability due to the action of the flow.

\subsection{Full aeroelastic bridge models vs. sectional models}

A pertinent issue for researchers planning wind tunnel tests of long-span bridges is the choice between a full aeroelastic model of the bridge and a sectional model of the deck [37]. In the former, besides the full three-dimensional geometry of the bridge, the dynamic attributes of the several components must also be adequately reproduced in order to obtain a valid global response of the bridge as a whole structure and of the interaction between its structural members. For large-span bridges, full model aeroelastic studies are undertaken because of the important role of towers and cables in the overall bridge response. Nowadays, large wind tunnels exist that can accommodate this type of studies, although at the expense of smaller scales of the model.

This is not the aim of sectional model tests, where the objective is rather studying the dynamic stability of the section proposed for a bridge. Thus, a sectional model reproduces a representative section of the bridge's deck, which is extruded over a length, to produce a twodimensional body for tests in the wind tunnel. For this purpose, the dynamic properties of the sectional model must be judiciously chosen.

Full aeroelastic model tests are usually conducted taking into consideration the effects of the terrain on the wind, whereas sectional models are usually tested in smooth flow. Therefore, in full model tests, the turbulence response is obtained directly and the effects of turbulence on flutter and vortex shedding are intrinsic and all three-dimensional aeroelastic effects are modelled implicitly. However, 
compared to sectional model tests, full three-dimensional tests involve higher cost and longer lead time; larger wind tunnels, which are costly to operate; and smaller scale of the model, which limits the level of detail of the flow that can be studied and possibly introduces Re dependency effects. Consequently, full aeroelastic model tests are often complemented with sectional model tests, as was the case in the design stage of the Trans-Tokyo Bay Bridge [38], to give an example. An interesting discussion of advantages and limitations of sectional models, taut-strip models, and full bridge models is given in [29]. Alternatively, instead of the full bridge, parts of the bridge, retaining some three-dimensional aspects, may be tested throughout the design phase; for example, at a certain point of the Messina Bridge Project, just the main span was tested at 1:250 scale.

The test of sectional models has been widely understood as a good tool to predict critical flutter instability as well as critical vortex-induced vibration. This is particularly accurate for box-girder and plate girder decks, when sectional model testing appears to predict full scale vortex shedding excitation, and there is evidence that turbulence has only but a minor effect on critical flutter speeds.

\subsection{Instrumentation}

To study in the wind tunnel the susceptibility of a bridge to aerodynamic instabilities, the model has to be free to oscillate in response to the action of the flow. This is a completely different situation of static testing. In what concerns sectional models, there are a few solutions, along with the associated instrumentation, to adequately support them in the wind tunnel. Typical suspension systems are based on helical springs. One configuration (used by e.g., [39]) is based on a single pair of suspension columns, which support the extremities of a shaft on the sectional model's axis of rotation. Other sets of helical springs, not contributing to the suspension though, can govern the torsional stiffness, and the setup also includes means of preventing rolling and horizontal displacements of the model. Another configuration employs four suspension columns to support the sectional model through two horizontal arms, one at each end of the model. The four columns tackle both torsional and flexural stiffness. Each suspension column includes two helical springs, one above and one below the suspension arm. Two horizontal wires limit the translation of the model in the horizontal plane. The entire assembly remains outside the test chamber. Systems of this type have been used, for example, by Larsen and Wall [40], and by the present authors [41].

Various types of sensors may be used with the suspension systems to monitor the windinduced motions of the deck model: laser reflexion sensors, piezoelectric accelerometers, or ring strain sensors [41].

The dynamics of the flow pattern around the model can be understood using diagnostic techniques such as laser sheet visualisation (LSV) [40]. Computational fluid dynamics (CFD), to be discussed in the next section, is becoming more often used also for this purpose, and the term CFD visualisation has been employed (e.g., [10, 42]). The classic techniques of smoke lines and wool tufts are still valuable for expedite assessments.

Another possibility is the simultaneous measurement of turbulent pressure fluctuations at multiple points on the surface of the model (e.g., [43]). By analysing the spectra correlation, the development and movement of recirculation bubbles can be understood. The interest in 
this technique has increased at the same rate as the increase of computational power, and, nowadays, the required post-processing is done swiftly. The duration of a wind tunnel test campaign using this technique has been reduced, making them more affordable.

\section{Numerical modelling}

\subsection{Introductory notes}

At present, three-dimensional unsteady numerical simulations where all the relevant physics of the interaction of turbulent wind with a flexible structure are completely described still involve a considerable investment of time, despite the steady increase in computational power over time. The case becomes even more difficult if wind gusts, surrounding complex terrain, or coupling with neighbour bridges, are to be included. Therefore, numerical simulations are carried out to look into a certain aspect of the whole problem. Often, numerical simulations complement wind tunnel testing. The engineer has to integrate the several pieces of information to come up with a design that not only is robust from the wind action point of view but also complies with economic and structural constraints.

Generally speaking, structural aspects are studied with the finite element method (FEM), whereas aerodynamic aspects are studied with the finite volume method (FVM), or some other common method in computational fluid dynamics (CFD). It should be said that, recently, there have been advances towards using FEM for the study of the flow field.

In FEM, the structure of the bridge, or a part of it, is discretized into simple structural elements. Values of interest are then obtained at the ends (nodes) of the element, by solving the numerical model equations, and a weighting function is used within an element to obtain the values at intermediary positions on it. There is a wide variety of elements that may be used and a large choice of weighting functions ranging from simple to more elaborate and precise.

In CFD, the common approach is the Eulerian approach, in which the fluid around the deck, or pylons, or even the whole bridge, up to a long distance from the structure, is discretized into small control volumes for which transport equations (mass, momentum, turbulence, and scalars) are solved. Another approach is to employ meshless methods.

The challenge has been to couple these two tools to describe the coupling that exists in the real world between structure and wind, in order to be able to study aeroelastic phenomena.

\subsection{Modelling of the structural dynamics}

Finite element (FE) is the method of choice to model the structural dynamics of a bridge and can provide insight into its dynamic response, such as the main modes of oscillation and associated natural frequencies. This is especially important for bridges with low structural damping, as is the case of cable-stayed or suspension bridges. Among many studies, a few published examples on suspension bridges are The John A. Roebling Bridge in Kentucky, USA [44]; Tamar Bridge in Plymouth, UK [45]; and the Bosphorus Bridge and the Fatih Sultan Mehmet Bridge, in Istanbul, Turkey [46]. 
It should be noted that FE simulations are usually carried out for structural reasons, for example to assess the response to seismic or traffic loads, and therefore, its use in the assessment of the aerodynamic behaviour of the bridge $[29,47]$ is just another benefit to be collected from the effort put into carrying out the simulations.

Accompanying the advances in computing power, the dynamic analysis of bridges employs, nowadays, high-resolution three-dimensional FE modelling, encompassing thousands of finite elements. Among the various types of finite elements, the following should be highlighted: beam, shell and slab elements for deck and towers, and tension-only spar elements for cables. More information on the number and type of finite elements typically employed in FE analysis of bridges can be found in [46], where a number of case studies are presented.

\subsection{Modelling of the aerodynamics}

\subsubsection{Finite volume method}

Arbitrary flows of viscous fluids are described by the Navier-Stokes equations. The numerical solution of these nonlinear partial differential equations for turbulent flows is very difficult. The finite volume (FV) is the most common method used in the formulation of the numerical problem. The domain is divided into small volumes and the balance equations are solved for each, in an iterative procedure across the domain. However, the wide range of length scales imply the use of a very fine mesh up to a level that requires huge computational resources thus making direct numerical simulation (DNS) still infeasible for engineering problems. Another approach is to model the finer scales and to explicitly solve the larger turbulent scales. Yet, the resulting model, large eddy simulation (LES) [48], is still computationally intensive. For practical problems in engineering, less demanding approaches are used, in particular the one based on time-averaged equations (RANS) complemented with turbulence models. Popular turbulence models are the $(\kappa-\varepsilon)$ (suitable for regions away from walls), the $\kappa-\omega$ (developed to tackle near wall effects), and the Reynolds stress model (which includes equations to deal with turbulent fluctuations in the three spatial directions). The single-equation Spalart-Allmaras model is also interesting for being much less computationally expensive and nevertheless useful in the study of airflow around bridge decks [16].

The problem can be further simplified by reducing the analysis to the two-dimensional space and/or by considering the flow to be steady. In two-dimensional, steady simulations, a crosssection of the deck is studied under the action of lateral wind to identify the existence of recirculation bubbles, their mean dimension, idea of the aerodynamic coefficients $\left(C_{L^{\prime}} C_{D^{\prime}}\right.$ and $\left.C_{M}\right)$, and how all of these features vary with angle of attack of the wind. Uniform wind or with shear (simulating the atmospheric boundary layer) can be studied.

In turn, unsteady simulations allow studying the pattern of recirculation bubble formation and vortex shedding around the deck's contour and their effect on the variation in time of the aerodynamic coefficients [42]. The effect of wind gusts can also be studied.

By allowing the structure's section to move in the domain in response to the aerodynamic forces acting over its contour and introducing mesh adaption techniques [49], it is possible to study fluid structure interaction, though without the three-dimensionality associated with the oscillation modes of the whole bridge. 
Three-dimensional simulations are much more computationally intensive. An example of three-dimensional simulation is that of [43], in which LES has been used to obtain flutterderivatives of a deck sectional model.

\subsubsection{Discrete vortex method}

Based on the existing discrete vortex method, HonoréWalther and Larsen [50] developed a relatively fast numerical model well suited for the simulation of two-dimensional bluff-body flows such as around the cross-section of bridge decks. A great advantage of this numerical tool is that it is mesh free.

The method takes the vorticity transport equation, spit into an advection part and a diffusion part, with appropriate boundary conditions at the solid surface involving the surface vortex sheet concept. A Lagrangian approach is then used in which vortex particles introduced in the domain are followed. The diffusion part is tackled via random walks. A scheme for marching in time is obtained from a Euler integration.

The input to the numerical model is the deck cross-section discretised into vortex-panels, typically a few hundreds of them. The model outputs are time series of aerodynamic force and moment, maps of pressure distribution, and streamlines. Aerodynamic derivatives can also be obtained. Larsen and Walther have applied the model to bridge decks of long-span bridges for which wind tunnel data are available and found that the model made good to excellent predictions regarding drag coefficient, Strouhal number, critical velocity for the onset of torsional and coupled flutter, and onset of vortex-induced vibrations.

\subsubsection{Finite element method}

While in the past FEM would not be the method of choice for solving flow equations, because unlike the FVM it would not inherently satisfy the local mass and momentum conservation, more recent advances have overcome this [51]. The new finite element procedure for the solution of incompressible Navier-Stokes equations is referred to as flow condition-based interpolation (FCBI) finite element method.

Panneer Selvam et al. [52] have used FEM together with LES to model turbulence to simulate the flow around Great Belt East Bridge approach span. The high accuracy in approximating the convection term made it possible to use a relatively modest number of nodes. Long timesteps can be used if the grid can align with the flow. The results from the 2D simulations were in reasonable agreement with wind tunnel results.

\section{Attenuation of adverse aerodynamic phenomena}

To begin with, it should be noted that the aerodynamic study of a deck can only be initiated after the overall geometry and main dimensions of its section have been established. Thus, at that time, considerable effort has already been put in the bridge structural design. Therefore, to avoid delays and costs, the corrective modifications to be proposed in order to diminish the possibility of wind exciting a vibration mode, as inferred from wind tunnel tests, should 
not lead to considerable reformulations of the initial design of the bridge. As has already been mentioned, small geometrical modifications to the bridge can lead to substantial changes of the airflow pattern around the structure and thereby modify its aerodynamic behaviour. This effect can be exploited to overcome any undesirable aspect of the bridge's aerodynamic response. Accordingly, modifications to bridge decks often consist in the addition of aerodynamic appendages, judiciously shaped, dimensioned, and positioned, or in minor changes to the original geometry.

Fujino and Siringoringo [4] review aerodynamic countermeasures that have been used in truss and box-girder bridges built all over the world to avoid aerodynamic instabilities like galloping, torsional flutter, and vortex-induced vibration. The countermeasures are specific of the aerodynamic phenomenon to mitigate.

\subsection{Buffeting}

The response of long-span cable-supported bridges to buffeting can be controlled through the use of auxiliary damping devices called tuned mass dampers (TMD). These are based on the secondary inertial system principle and consist of a mass attached to the structure through a spring and a dashpot. A variation of TMD is the tuned liquid damper (TLD) where the mass is replaced by a liquid (usually water).

Other types of cost-efficient dampers for mitigating buffeting response have been developed, like the tuned liquid column damper (TLCD) and its variants. This concept, first proposed by Sakai et al. [53], consists of a U-tube container in which one or more sections are partially obstructed by a plate with an orifice at the centre. The energy of structural oscillation is dissipated by the combined action of the liquid inertia, weight, and the damping effect associated with hydraulic pressure loss through the orifices within the tube. In principle, TLCD can be tuned (or retuned) to the frequency of the structure by designing it with the appropriate wetted length, or "liquid length". The advantages of TLCD systems include low cost and low maintenance, while they also represent a water reservoir that can be used for fire-fighting.

An evolution with adaptive frequency tuning capacity is the semi-active TLCD, which features an air chamber at each end of the tube where the pressures are actively controlled. Shum et al. [54] numerically investigated the performance of this system for suppressing combined lateral and torsional vibration along the construction of a real long-span cable-stayed bridge and concluded that it can effectively reduce the buffeting response of the bridge for the various construction stages. The same authors investigated a further development of the concept encompassing multiple pressurised TLCD, which provides more flexibility in the system design.

\subsection{Vortex-induced vibrations}

As previously mentioned, VIV is an aeroelastic phenomenon that occurs at low wind speeds and that is strongly related to the bridge section's shape. When designing the section's shape, there must be an effort towards minimising the possibility of occurrence of these phenomena $[26,40]$, since, generally, any ad-hoc solutions will increase the complexity of the structure 
and its construction and maintenance costs. If despite this effort, it is not possible to guarantee the absence of VIV, then other countermeasures have to be adopted.

One such countermeasure is the installation of tuned mass dampers (TMD), which are a secondary vibration system designed to have the same natural frequency of the main vibration system that it intends to cancel (i.e., the bridge). When the bridge oscillates, the secondary system acts as a stabiliser because of the action of its damping force on the main vibration system. However, the installation of TMD requires the existence of considerable free space in the bridge deck, which is not always available.

Over the past decades, various studies demonstrated that aerodynamic appendages, like double-flaps, flaps, or skirts, are effective countermeasures against VIV. These attachments inhibit the formation of the vortices responsible for VIV by directing high-speed flow to sweep the relevant deck's surfaces; moreover, the appendages themselves generate turbulence close to the surface of the bridge, which improves resistance to flow separation.

Other countermeasures have been proposed, like guide vanes, which have been employed for example on the Great Belt Suspension Bridge, as already mentioned in the Introduction.

\subsection{Aeroelastic instabilities}

Since the failure of the Original Tacoma Narrows Bridge, mainly two types of deck girders have been promoted: the streamlined box-girder and the truss stiffened girder. Wind tunnel tests came to reveal that the use of either one was not an absolute assurance of absence of flutter instabilities, and hence other countermeasures were developed.

For the truss stiffened girder deck, one countermeasure generally used is the installation of gratings on the girder, which reduces the pressure difference between the lower and the upper surfaces of the deck and thus settles flutter. This solution was adopted in the suspension bridges over the Akashi Strait in Japan and over the Tagus River in Portugal, with central spans of 1990 and 1013 m, respectively.

Flutter instabilities in plate girder decks can also be controlled with gratings. The influence of the opening ratio and the location of the grating have been object of study.

In regards to streamlined box-girders, studies have shown that the aerodynamic stability can be improved by allowing for a central slot in the box section. This increases the critical flutter wind speed of the bridge, and it further rises with the slot's width [24].

Numerous other solutions have been used to satisfy bridge construction codes' requirements for flutter. Some of them are triangular fairings [21, 26], active flaps [55], and passive flaps [56].

Galloping can particularly affect very slender box-girders. It may be difficult to control galloping through the increment of structural damping because of the small effect on the critical wind speed and given the diverging nature of the oscillation's amplitude. The countermeasure usually adopted to minimise the effects of galloping is the attachment of relatively small horizontal plates to the surface of the lower part of the girder. 


\section{Example of bridge design aided by wind tunnel studies: the Vila- Real Bridge}

We have seen that, in what concerns to bridges, Codes of Practice strongly encourage wind tunnel tests for cases encompassing long span or great heights. If susceptibility to adverse aerodynamic phenomena is identified in the tests, then the solutions discussed in the previous section can be considered. The economics of the project favours minor geometric changes (from the structural viewpoint) comparatively to the addition of aerodynamic appendages. Therefore, wind tunnel tests and structural design should be coordinated so that any geometrical modification possibly proposed can be reflected in the final design.

In this section, the study of the cable-stayed Vila-Real Bridge, over the Corgo River Valley, Portugal, is presented as an example of the aid wind tunnel tests can provide in the design of the deck cross-section. The tests were carried out by the authors and associated team at the Laboratory for Fluid Dynamics and Applied Thermodynamics of the Department of Mechanical and Industrial Engineering (DEMI) of Universidade Nova de Lisboa (UNL).

The deck entails a concrete single-cell box-girder with the top flange extended and supported by inclined struts, also in concrete. It is a bridge cable-stayed in the midplane, and its importance among this family of bridges is made clear in Figure 5. Given its main span of $300 \mathrm{~m}$ and deck height of $230 \mathrm{~m}$ above the river, wind tunnel tests were due, as pointed out in Section 3 (Codes of Practice).

When the overall geometry and main dimensions of the deck section had been established, by the engineering office in charge of the bridge structural design, it was time to address, through wind tunnel tests, the susceptibility of the bridge to aeroelastic instabilities. After a small survey of aerodynamic laboratories, the study was assigned to our laboratory. Over the years, the laboratory has acquired a reasonable experience in static tests of civil engineering structures, namely viaducts, pylons of stay-bridges, telecommunication towers, and concrete poles for overhead electrical transmission lines. The purpose of the study was to examine the

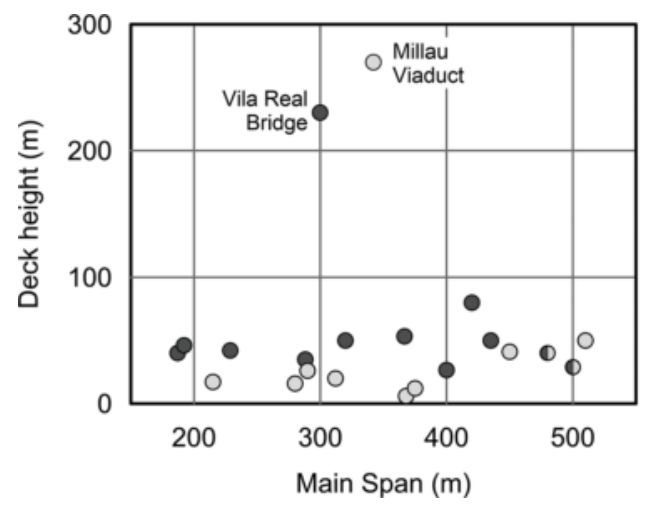

Bridges cable-stayed in the central plane. In increasing span order:

- Concrete: Veterans' Glass City Skyway (US) Varina-Enon (US) Chesapeake \& Delawa
Canal (US) Coatzacoalcos II (MX) Vila Real (PT) Brotonne (FR) Sunshine Skyway (US) I'Iroise (FR) Centennial (PA) Bãi Cháy (VN)
O Steel: Shin-Onomichi (JP) Bonn Nord (DE) Farø South Bridge (DK) Vanšu (LV) Millau (FR) Düsseldorf-Fiehe (DE) Solidarity (PL) Rama IX (TH) Tsurumi Tsubasa (JP)

- Composite: Geo-Geum 2 (KR) Sungai Johor (MY)

Figure 5. Deck height versus main span (adapted from [10]). 
bridge's dynamic response to the action of lateral wind, for the construction and operation configurations, and to propose attenuation measures if found necessary.

Prior to wind tunnel tests, in order to understand the way the wind approaches the Corgo Valley, numerical simulations of the wind over terrain were performed with input from statistical wind data from a meteorological station a few kilometres far off.

For the reasons stated in Section 4.3, the tests were conducted with a sectional model of the deck supported in the wind tunnel by an elastic suspension system. The model, at a scale of 1:66.7, has a skeleton, of aluminium frames mounted on four steel bars, that confers the indispensable stiffness for it to not bend or twist in the dynamic tests, as well as brings the weight to the required range. The shape of the box-girder and top flange was then obtained with balsa wood lined with thin birch plywood, leaving exposed the portion of the aluminium frames that represent the inclined struts.

The suspension system, already referred to in Section 4.4 and described in detail in [41], was adjusted taking into consideration the suspended mass, the logarithmic decrement factor of the combined suspension assembly and model, and the ratio of torsional to heaving natural frequencies of the bridge that were made available by the design team from FE simulations. The adjustment was made for each configuration: bare deck during construction (with the underlying Eigen frequencies corresponding to the situation in which the bridge's central span is about to be closed) and fully equipped deck (with frequencies corresponding to a continuous construction over the whole length of the bridge).

The proper combination of forces inferred from the measurements of six ring strain sensors present in the assembly produces the dynamic action of the flow on the model in terms of lift, drag, and roll and pitch moments. The model was tested for various angles of attack and the RMS of the amplitude of oscillations both in torsion and vertical translation was obtained for the range of reduced velocities of practical interest.

Since the tests were conducted in smooth flow, buffeting shows up just slightly in the results and is possibly related to the vortex field generated by flow separations along the contour of the deck. Diverging amplitude, which would correspond to flutter, has never been found. The results for the construction configuration exhibit no peaks whatsoever, while those for the operation configuration show peaks indicating possible susceptibility to VIV. Figure 6 shows a sample of results (angle of attack $\alpha=+2^{\circ}$ ). It should be pointed out that the model is more sensitive to oscillation than the real deck since, in strict terms, the sectional model represents a deck of infinite span.
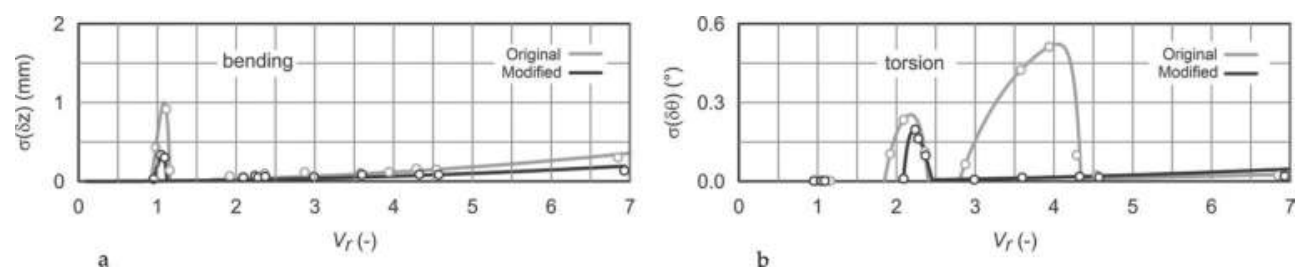

Figure 6. Results for fully equipped deck in the original and modified configurations at an angle of attack $\alpha=+2^{\circ}$. Amplitudes of: (a) vertical oscillations; (b) torsional oscillations. 
To gain insight into the flow topology, two-dimensional numerical simulations of the flow were carried out, employing unsteady Reynolds-averaged Navier-Stokes (URANS) equations. By comparing comprehensively, the numerical and experimental results, it was possible to understand that, at least for this particular deck section, VIV occurs whenever the flow pattern displays simultaneously the following two features (Figure 7): (a) above the deck, a large coherent vortex structure on the windward part or irregular flow anywhere; (b) below the box-girder base, separated flow without reattachment. This led to the conclusion that to eliminate, or at least attenuate, the oscillatory movements of the model at certain wind speeds it would suffice to prevent vortex-shedding from at least one side of deck.

To reduce the dimensions of the recirculation bubble below the box-girder, whenever it was formed, in order to facilitate its reattachment and thus prevent vortex-shedding, the edges at the base of the box-girder were rounded. The border beam profile was also rounded. Wind tunnel tests of the deck with these slight geometric modifications showed that they were beneficial.

The geometric modifications were then proposed to the engineering office undertaking the bridge design and a meticulous discussion followed to define the transposition of the modifications to the full-scale bridge. They are visible in Figure 8, and the dashed lines in the sketches therein refer to the original contour.
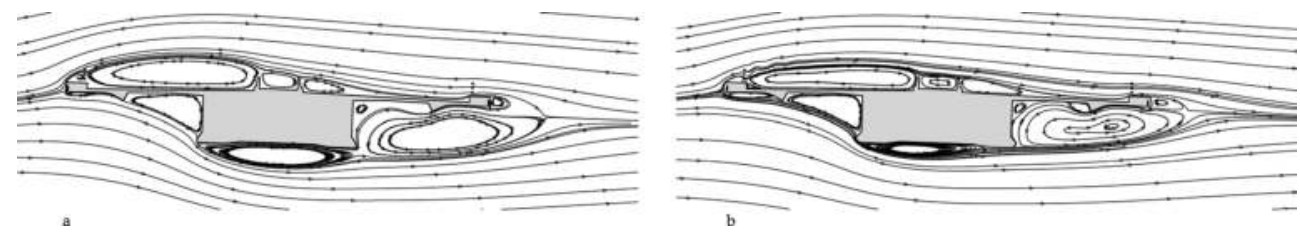

Figure 7. Streamlines from numerical simulation for fully equipped deck at $\alpha=+2^{\circ}$, in configurations: (a) original, showing a large recirculation bubble over the deck, on the windward part, and separated flow without reattachment below the box-girder base; (b) modified, showing thinner bubbles and reattached flow below the girder (from [10]).
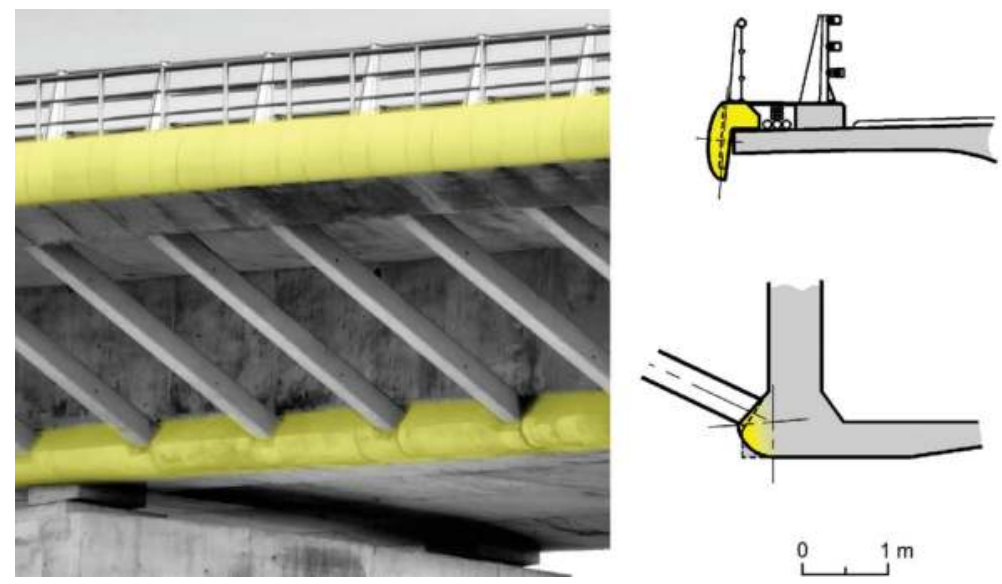

Figure 8. Photo and schemes of details of the Vila-Real Bridge deck, showing the modifications introduced by the aerodynamic study (original photo courtesy of Prof. A.P.V. Urgueira, Universidade Nova de Lisboa). 
As can be seen in the results of Figure 6, the modifications introduced completely eliminated the prominent peak in torsional oscillations and reduced the other minor peaks (both in vertical translation and in torsion). The results in Figure $6 \mathbf{a}$ also suggest a reduction of what is one of the forms of buffeting.

\section{Conclusion}

The execution of projects of special structures is always complex and requires contributions from different branches of engineering. It is important that the structural design team is complemented, in due time, by aerodynamic specialists with the competence and access to the means to perform the required special studies. The Vila-Real Bridge case that has been discussed here is an example of how laboratorial means were made available, of the capacity of timely response, and of how modifications to improve the aerodynamic performance of a bridge deck can turn out to be very subtle from the geometric point of view (or even favour the aesthetics of the bridge) while being very effective with regards to aerodynamics.

It should be pointed out that major structures that very exposed to wind action are the ones that justify wind tunnel studies, and the costs of the aerodynamic studies are always a small parcel of the total investment for this kind of projects.

\section{Acknowledgements}

This work has been funded by Fundação para a Ciência e Tecnologia (PEst-OE/EME/ UI0667/2014).

\section{Author details}

Daniel C. Vaz*, Raquel A.B. Almeida and António R. Janeiro Borges

*Address all correspondence to: dv@fct.unl.pt

UNIDEMI and DEMI, Universidade Nova de Lisboa, Caparica, Portugal

\section{References}

[1] Battista RC. Reduction of vortex-induced oscillations of Rio-Niterói bridge by dynamic control devices. Journal of Wind Engineering and Industrial Aerodynamics. 2000;84(3):273288. DOI: 10.1016/S0167-6105(99)00108-7

[2] Larsen A, Esdahl S, Andersen JE, Vejrum T. Storebælt suspension bridge - Vortex shedding excitation and mitigation by guide vanes. Journal of Wind Engineering and Industrial Aerodynamics. 2000;88(2):283-296. DOI: 10.1016/S0167-6105(00)00054-4 
[3] Weber F, Maślanka M. Precise stiffness and damping emulation with MR dampers and its application to semi-active tuned mass dampers of Wolgograd bridge. Smart Materials and Structures. 2014;23(1). DOI: 10.1088/0964-1726/23/1/015019

[4] Fujino Y, Siringoringo D. Vibration mechanisms and controls of long-span bridges. Structural Engineering International, The International Association for Bridge and Structural Engineering (IABSE). 2013;23(3):248-268. DOI: 10.2749/101686613X13439149156886

[5] Irwin HPA. Wind Tunnel and Analytical Investigations of the Response of Lion's Gate Bridge to a Turbulent Wind. Ottawa, Canada: National Aeronautical Establishment; 1977

[6] Davenport AG. The response of slender, line-like structures to a gusty wind. Proceedings of the Institution of Civil Engineers. 1962;23(3):389-408. DOI: 10.1680/iicep.1962.10876

[7] Larsen A, Larose GL. Dynamic wind effects on suspension and cable-stayed bridges. Journal of Sound and Vibration. 2015;334:2-28. DOI: 10.1016/j.jsv.2014.06.009

[8] EN 1991-1-4:2005. Eurocode 1: Actions on Structures - Part 1-4: General actions - Wind Actions. European Committee for Standardization; 2005

[9] $\mathrm{Wu} \mathrm{T}$, Kareem A. An overview of vortex-induced vibration (VIV) of bridge decks. Front Struct. Civil Engineering. 2012;6(4):335-347. DOI: 10.1007/s11709-012-0179-1

[10] Vaz DC, Almeida RAB, Didier E, Urgueira APV, Borges ARJ. Improving the aerodynamic performance of Vila-Real bridge deck-section. Journal of Wind Engineering and Industrial Aerodynamics. 2016;156(Supplement C):72-83. DOI: 10.1016/j.jweia.2016.07.002

[11] Den Hartog JP. Mechanical Vibrations. New York: McGraw-Hill Book Company; 1956

[12] Theodorsen T. General Theory of Aerodynamic Instability and the Mechanism of Flutter; 1934. DOI: https://ntrs.nasa.gov/search.jsp?R=19930090935

[13] Technical Committee 12 - Wind. Recommendations for Calculating the Effects of Wind on Constructions. 2nd ed. European Convention for Constructional Steelwork (ECCS); 1987

[14] Scanlan RH, Sabzevari A. Experimental aerodynamic coefficients in the analytical study of suspension bridge flutter. Journal of Mechanical Engineering Science. 1969;11(3):234242. DOI: 10.1243/JMES_JOUR_1969_011_031_02

[15] Bartoli G, Mannini C. A simplified approach to bridge deck flutter. Journal of Wind Engineering and Industrial Aerodynamics. 2008;96(2):229-256. DOI: 10.1016/j.jweia.2007. 06.001

[16] Vaz DC, Didier E, Borges ARJ. Effects of geometry modification on the aero-dynamics of a generic bridge deck section. In, JCF Pereira and A. Sequeira (Eds.): ECCOMAS CFD 2010; 2010; Lisbon

[17] Miyata T. Historical view of long-span bridge aerodynamics. Journal of Wind Engineering and Industrial Aerodynamics. 2003;91(12):1393-1410. DOI: 10.1016/j.jweia.2003.09.033

[18] Saito T, Sakata H. Aerodynamic stability of long-span box girder bridges and anti-vibration design considerations. Journal of Fluids and Structures. 1999;13(7):999-1016. DOI: 10.1006/jfls.1999.0238 
[19] Bienkiewicz B. Wind-tunnel study of effects of geometry modification on aerodynamics of a cable-stayed bridge deck. Journal of Wind Engineering and Industrial Aerodynamics. 1987;26(3):325-339. DOI: 10.1016/0167-6105(87)90003-1

[20] Bruno L, Mancini G. Importance of deck details in bridge aerodynamics. Structural Engineering International. 2002;5:289-294. DOI: 10.2749/101686602777965234

[21] Wang Q, Liao H, Li M, Xian R. Wind tunnel study on aerodynamic optimization of suspension bridge deck based on flutter stability. In: The 7th Asia-Pacific Conference on Wind Engineering (APCWE-VII); Taipei, Taiwan; 2009

[22] Zhengqing $\mathrm{C}$, Xugang H, Keijian O. A study of effect of cable aerodynamic forces and deck static deformation on flutter for cable-stayed bridges. In: The 5th International Symposium on Computational Wind Engineering (CWE2010); North Carolina, USA: Chapel Hill; 2010

[23] Larsen A. Advances in aeroelastic analyses of suspension and cable-stayed bridges. Journal of Wind Engineering and Industrial Aerodynamics. 1998;74(Supplement C): 73-90. DOI: 10.1016/S0167-6105(98)00007-5

[24] Sato H, Kusuhara S, Ogi K, Matsufuji H. Aerodynamic characteristics of super long-span bridges with slotted box girder. Journal of Wind Engineering and Industrial Aerodynamics. 2000;88(2):297-306. DOI: 10.1016/S0167-6105(00)00055-6

[25] Larsen A. Aerodynamic stability and vortex shedding excitation of suspension bridges. In: 4th International Conference on Advances in Wind and Structures (AWAS'08). Jeju, Korea: Techno Press; 2008

[26] Wang Q, Liao H, Li M, Ma C. Influence of aerodynamic configuration of a streamline box girder on bridge flutter and vortex-induced vibration. Journal of Modern Transportation. 2011;19(4):261-267. DOI: 10.1007/BF03325767

[27] COWI-“Bridge Aerodynamics", retrieved from: http://www.cowi.com/menu/service/ bridgetunnelandmarinestructures/bridges/bridge-dynamics/aerodynamics/documents/ 021-1700-016e_09a_aerodynamics_low.pdf

[28] Diana G, Fiammenghi G, Belloli M, Rocchi D. Wind tunnel tests and numerical approach for long span bridges: The Messina bridge. Journal of Wind Engineering and Industrial Aerodynamics. 2013;122(Supplement C):38-49. DOI: 10.1016/j.jweia.2013.07.012

[29] Larsen A. Aerodynamic aspects of the final design of the $1624 \mathrm{~m}$ suspension bridge across the Great Belt. Journal of Wind Engineering and Industrial Aerodynamics. 1993;48(2): 261-285. DOI: 10.1016/0167-6105(93)90141-A

[30] Specifications for Highway Bridges, Japan Road Association

[31] Design Standards of Superstructures for Long-Span Bridges. Honshu-Shikoku Bridge Authority

[32] "LRFD Bridge Design Specifications" by the American Association of State Highway and Transportation Officials 
[33] Wind-Resistant Design Specification for Highway Bridges (JTG/TXX-2004) (in Chinese). Beijing: People's Communication Press; 2004

[34] CAN/CSA-S6-00 (R2005) Canadian Highway Bridge Design Code

[35] Australian Standard AS 5100.2-2004, Bridge design Part 2: Design loads, section 16 - Wind loads

[36] BS 5400, National Standards Body, UK

[37] Wardlaw RL. Sectional versus full model wind tunnel testing of bridge road decks. Proceedings of the Indian Academy of Sciences Section C: Engineering Sciences. 1980;3(3): 177-198. DOI: 10.1007/BF02861559

[38] Fujino Y, Yoshioka Y. Wind-induced vibration and control of trans-Tokyo Bay crossing bridge. Journal of Structural Engineering, ASCE. 2002;128(8):1012-1025. DOI: 10.1061/ (ASCE)0733-9445(2002)128:8(1012)

[39] Manzoor S, Hémon P, Amandolese X. On the aeroelastic transient behaviour of a streamlined bridge deck section in a wind tunnel. Journal of Fluids and Structures. 2011;27(8):1216-1227. DOI: 10.1016/j.jfluidstructs.2011.07.003

[40] Larsen A, Wall A. Shaping of bridge box girders to avoid vortex shedding response. Journal of Wind Engineering and Industrial Aerodynamics. 2012;104(Supplement C): 159-165. DOI: 10.1016/j.jweia.2012.04.018

[41] Almeida RAB, Vaz DC, Urgueira APV, Janeiro Borges AR. Using ring strain sensors to measure dynamic forces in wind-tunnel testing. Sensors and Actuators A: Physical. 2012;185(Supplement C):44-52. DOI: 10.1016/j.sna.2012.07.024

[42] Mannini C, Sbragi G, Schewe G. Analysis of self-excited forces for a box-girder bridge deck through unsteady RANS simulations. Journal of Fluids and Structures. 2016;63 (Supplement C):57-76. DOI: 10.1016/j.jfluidstructs.2016.02.007

[43] Anina Š, Rüdiger H, Stanko B. Numerical simulations and experimental validations of force coefficients and flutter derivatives of a bridge deck. Journal of Wind Engineering and Industrial Aerodynamics. 2015;144(Supplement C):172-182. DOI: 10.1016/j.jweia.2015.04.017

[44] Ren W-X, Blandford G, Asce M, Harik I. Roebling suspension bridge. I: Finite-element model and free vibration response. Journal of Bridge Engineering. 2004;9(2):110-118. DOI: 10.1061/(ASCE)1084-0702(2004)9:2(110)

[45] Westgate RJ, Brownjohn JMW. Development of a tamar bridge finite element model. In: Dynamics of Bridges. In: Volume 5. Conference Proceedings of the Society for Experimental Mechanics Series; New York, NY: Springer; 2011. pp. 13-20. DOI: 10.1007/ 978-1-4419-9825-5_2

[46] Kilic SA, Raatschen HJ, Körfgen B, Apaydin NM, Astaneh-Asl A. FE model of the Fatih Sultan Mehmet suspension bridge using thin Shell finite elements. Arabian Journal for Science and Engineering. 2017;42(3):1103-1116. DOI: 10.1007/s13369-016-2316-y 
[47] Barata V. Viaduto do Corgo da A.E. Transmontana. In: Proceedings of the Encontro Nacional Betão Estrutural (in Portuguese); Faculdade de Engenharia da Universidade do Porto-FEUP. 2012. pp. 1-20

[48] Sarwar MW, Ishihara T, Shimada K, Yamasaki Y, Ikeda T. Prediction of aerodynamic characteristics of a box girder bridge section using the LES turbulence model. Journal of Wind Engineering and Industrial Aerodynamics. 2008;96(10):1895-1911. DOI: 10.1016/j. jweia.2008.02.015

[49] Scotta R, Lazzari M, Stecca E, Cotela J, Rossi R. Numerical wind tunnel for aerodynamic and aeroelastic characterization of bridge deck sections. Computers and Structures. 2016;167(Supplement C):96-114. DOI: 10.1016/j.compstruc.2016.01.012

[50] HonoréWalther J, Larsen A. Two dimensional discrete vortex method for application to bluff body aerodynamics. Journal of Wind Engineering and Industrial Aerodynamics. 1997;67-68(Supplement C):183-193. DOI: 10.1016/S0167-6105(97)00072-X

[51] Kohno H, Bathe KJ. Insight into the flow-condition-based interpolation finite element approach: Solution of steady-state advection-diffusion problems. International Journal for Numerical Methods in Engineering. 2005;63(2):197-217. DOI: 10.1002/nme.1276

[52] Panneer Selvam R, Tarini MJ, Larsen A. Computer modelling of flow around bridges using LES and FEM. Journal of Wind Engineering and Industrial Aerodynamics. 1998;77(Supplement C):643-651. DOI: 10.1016/S0167-6105(98)00179-2

[53] Sakai F, Takaeda S, Tamaki T. Tuned liquid column damper-new type device for suppression of building vibration. In: Proceedings of 1st International Conference on HighRise Buildings; Nanjing, China; 1989. pp. 926-931

[54] Shum KM, YL X, Guo WH. Buffeting response control of a long span cable-stayed bridge during construction using semi-active tuned liquid column dampers. Wind and Structures. 2006;9:271-296. DOI: 10.12989/was.2006.9.4.271

[55] Nissen HD, Sørensen PH, Jannerup O. Active aerodynamic stabilisation of long suspension bridges. Journal of Wind Engineering and Industrial Aerodynamics. 2004;92(10):829847. DOI: 10.1016/j.jweia.2004.03.012

[56] Phan D-H, Nguyen N. Flutter and buffeting control of long-span suspension bridge by passive flaps: Experiment and numerical simulation. International Journal of Aeronautical and Space Sciences. 2013;14(1):46-57. DOI: 10.IJASS.2013.14.1.46 
